\title{
The production of live attenuated influenza $A$ strains by recombination with A/Okuda/57 $\left(\mathbf{H}_{2} \mathbf{N}_{2}\right)$
}

\author{
D. MCCAHON* \\ B.Sc., Ph.D. \\ A. S. BEARE $\dagger$ \\ M.D., F.R.C.Path. \\ V. STEALEY
B.Sc., Ph.D.
}

Virology Department, Wellcome Research Laboratories, Beckenham, Kent, and

† Clinical Research Centre, Harvard Hospital, Salisbury, Wilts

\begin{abstract}
Summary
A new recombination system for the production of live attenuated influenza-A vaccine strains is described. The attenuated parent is the Japanese strain A/Okuda/ $57\left(\mathrm{H}_{2} \mathrm{~N}_{2}\right)$ which has a known passage history and a good record of human safety. This virus has been recombined with two recent field isolates, A/England/ 42/72 and $A /$ Finland/4/74, and in each case several recombinants were isolated and tested in volunteers. These recombinants differed in their degree of attenuation, but on each occasion it was possible to choose one that was suitably attenuated for a candidate vaccine strain. The genetic stability of such a recombinant produced with the A/England/42/72 strain was demonstrated by subjecting it to three successive human passages. A single intra-nasal dose of the A/Finland/ $4 / 74$ recombinant vaccine gave a seroconversion rate of $75 \%$.
\end{abstract}

This recombination system offers considerable advantages for the production of live influenza virus vaccines.

\section{Introduction}

In a previous report (McCahon et al., 1973) the transfer of attenuation for man from the Mount Sinai strain of A/Puerto Rico/8/34 $\left(\mathrm{H}_{0} \mathrm{~N}_{1}\right)$ to the virulent strain, A/England/939/69 $\left(\mathrm{H}_{3} \mathrm{~N}_{2}\right)$ was described. However, we now believe that the A/PR/8/34 strain is unsuitable for general use as a donor of attenuation for three reasons: (1) its passage history is incomplete, and what is known (e.g. Maassab, Kendal and Davenport, 1972) shows that there has been ample opportunity for possible genetic interactions with viruses which are completely undesirable in a live human vaccine; (2) few of the recombinants are sufficiently attenuated for immediate use; with A/England/939/69 as the virulent parent virus, two of the four recombinants tested were attenuated (Beare and Hall, 1971); with A/England/42/72 as

\footnotetext{
* Present address: Genetics, Animal Virus Research Institute, Pirbright, Surrey.
}

the virulent parent virus, two of the three recombinants tested were attenuated; and with $\mathrm{A} /$ Port Chalmers/1/73 as the virulent parent virus, neither of the two recombinant viruses tested were attenuated (Beare and Schild, unpublished results). In fact, with the 1972 and 1973 recombinants, it was considered desirable to select horse serum-resistant mutants as a further attenuation step (Huygelen et al., 1973; Peetermans, Lamy and Delem, 1974); (3) its antigens are remote from recent experience in the population. Influenza recombinants have been made in many different laboratories for many years and we know of no evidence that a properly cloned virus has been unstable or contained an abnormal genetic complement. However, should it happen that a recombinant vaccine strain were to 'disassociate' to its parent viruses it would be preferable that there should be a reasonable level of antibody to the attenuated parent virus in the human population.

In this paper we describe the use of a Japanese strain, A/Okuda/57 $\mathrm{H}_{2} \mathrm{~N}_{2}$, that is believed to be preferable to other available parents. Its passage history is known, there is good evidence for its safety in man and it has been possible to transfer its attenuation at high frequency to two recent strains, A/England/42/72 and A/Finland/4/74.

Materials and methods

\section{Parent viruses}

AlOkuda/57 ( $\mathrm{H}_{2} \mathrm{~N}_{2}$, Okuno strain). This virus was isolated in eggs in 1957 from a patient called Okuda in Osaka, Japan (Nakamura, 1961), and since that time has been passaged only in eggs. It has been tested for attenuation at various passage levels and especially after 273 or more passages, it seemed completely apathogenic for man. A freeze-dried sample of 280th egg passage material (Lot No. 15, 25 September, 1967) was obtained from Professor Y. Okuno, Osaka University, Japan, via Dr D. A. J. Tyrrell. The virus was passed once in specific pathogen-free (SPF) eggs before recombination with 
A/England/42/72, but was cloned twice at limit dilution in SPF eggs, as described below, before recombination with $\mathrm{A} /$ Finland/4/74.

A/England/42/72 $\left(\mathrm{H}_{3} \mathrm{~N}_{2}\right)$. This virus had received a total of three egg passages since its original isolation in eggs and had not been grown in any other tissues. It was obtained as a freeze-dried sample from $\mathrm{Dr}$ M. S. Pereira of the Public Health Laboratory, Colindale. Before recombination it was passed once in the allantoic cavity of 10-day-old SPF eggs.

A/Finland/4/74 $\left(\mathrm{H}_{3} \mathrm{~N}_{2}\right)$. This virus was obtained as a throat swab from Dr K. Cantell of the Serum Institute, Helsinki. It was cloned twice at limit dilution in 10- or 11-day-old SPF eggs before recombination.

\section{Virus titration}

Virus was titrated in 10- or 11-day-old embryonated eggs in allantois-on-shell (AOS) cultures derived from such eggs (Fazekas de St Groth and White, 1958) and incubated as described previously (McCahon et al., 1973). Four or five eggs or AOS cultures were used per $1 / 3 \cdot 2$ dilution and incubation was for 3 days at approximately $37^{\circ} \mathrm{C}$. Calculation of the titre was by the method of Reed and Muench (1938) and was expressed as $50 \%$ egg infectious doses $\left(\mathrm{EID}_{50}\right)$ or AOS-infectious doses (AOS-ID ${ }_{50}$ ) per $\mathrm{ml}$.

\section{Recombination system}

The two viruses were recombined in $24 \mathrm{~mm}^{2}$ AOS cultures as outlined in Fig. 1.

After infection the cultures were incubated at $37^{\circ} \mathrm{C}$ for $2-4 \mathrm{hr}$, and then rabbit antiserum prepared against purified A/Singapore/57 $\left(\mathrm{H}_{2} \mathrm{~N}_{2}\right)$ was added to each culture at a dilution (1/1000) sufficient to suppress the growth of $\mathrm{A} / \mathrm{Okuda} / 57$ in $90 \%$ of cultures.

After a total incubation period of 18-24 hr, $0 \cdot 25$ $\mathrm{ml}$ of a $0.5 \%$ sterile suspension of SPF fowl erythrocytes was added to each AOS culture. The presence of haemagglutination was taken as an indication of a significant degree of virus growth. Under our conditions, control cultures infected only with A/Okuda/ 57 showed no haemagglutination, but $25 \%$ of control cultures infected only with the virulent parent showed weak haemagglutination. The medium with added erythrocytes from presumptive recombinant-containing cultures was collected and stored at $-70^{\circ} \mathrm{C}$ until cloned by the procedure described previously (McCahon et al., 1973).

\section{Growth test}

In this test, 10- or 11-day-old eggs were inoculated by the allantoic sac route, using between three and ten eggs for each isolate. The two parent viruses were included in each test. After 18-54 hr incubation at $37^{\circ} \mathrm{C}$, the yield of haemagglutinating units (HAu)

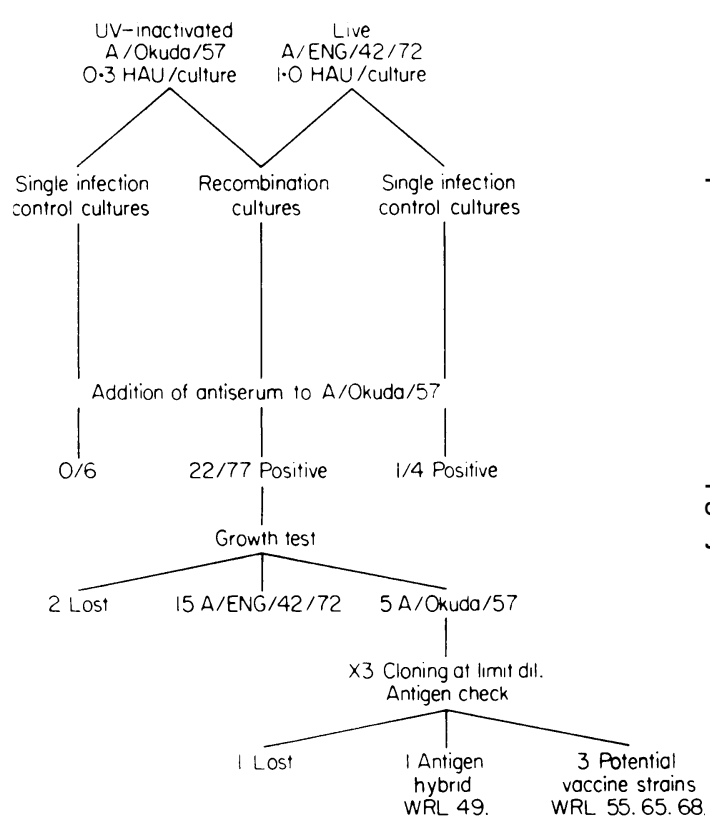

FIG. 1. Production, isolation and cloning of Okuda recombinants.

per egg was assayed (macro haemagglutination tes $0.25 \mathrm{ml}$ two-fold dilutions of virus). There usually a clear difference in the yields of the two parent viruses (A/Okuda/57, average yield $512 \mathrm{O}$ $\mathrm{HAu} / 0.25 \mathrm{ml} ; \mathrm{A} /$ England $/ 42 / 72$, average yield 1280 $\mathrm{HAu} / 0.25 \mathrm{ml} ; \mathrm{A} /$ Finland $/ 4 / 74$, average yield 1280 $\mathrm{HAu} / 0.25 \mathrm{ml}$ ), while the recombinant viruses pro- $\overrightarrow{\overrightarrow{0}}$ duced as much as or slightly less haemagglutinin 3 than the A/Okuda/57 parent virus.

In some experiments, the yields of infectious virus were also measured. The relative amounts wereo essentially the same as in the haemagglutinin assay, namely A/Okuda/57 produced $10^{9 \cdot 8} \mathrm{AOS}-\mathrm{ID}_{50} / \mathrm{ml}$, 응 the virulent parents produced between $10^{7.0}$ and $10^{7.5}$ AOS $-\mathrm{ID}_{50} / \mathrm{ml}$, and the recombinants produced between $10^{8 \cdot 0}$ and $10^{8 \cdot 5}$ AOS- $I D_{50} / \mathrm{ml}$.

\section{Serological techniques}

These were performed as described previously? (McCahon et al., 1973).

\section{Examination of volunteers in isolation at Salisbury}

(a) Clinical surveillance. The system used has beenw described previously (Tyrrell, 1963; Beare et al., श्र 1971). Clinical reactions were graded as nil, mild, moderate or severe and a nil or mild reaction was regarded as acceptable for a vaccine strain. Moderate ${ }^{?}$ reactions were regarded as unacceptable unless they뭉 occurred in only an occasional individual. If $\mathrm{a}_{\mathbb{\mathrm { O }}}$ volunteer needed to rest in bed even for a short 
period, the reaction was graded as severe. A single severe reaction, if supported by a high clinical score, was considered as evidence that a virus was unsuitable as a live vaccine. The clinical score is a more objective and numerical measure of reactions. As a rough guide to the significance of the scores shown in Tables 1, 2 and 3, over the 2 years during which these recombinants and a few parent wild viruses were tested, 154 volunteers classed as having nil or mild clinical reactions had a mean clinical score of 4.4 (range $0-31$ ), ten volunteers with moderate clinical reactions had a mean clinical score of 32 (range 20-55) and eleven volunteers with severe clinical reactions had a mean clinical score of 57 (range 2890).

(b) Virus excretion. Nasal washings collected on the third and fourth days after inoculation were mixed with an equal quantity of nutrient broth and stored in aliquots at $-70^{\circ} \mathrm{C}$. Samples were tested for infectivity by inoculation into the allantoic sac of four 10- or 11-day-old embryonated eggs. The allantoic fluids were tested with $0.5 \%$ fowl erythrocytes for the presence of haemagglutinin after incubation at $37^{\circ} \mathrm{C}$ for $2-3$ days.

(c) Serology. Sera collected from volunteers before and 2-3 weeks after the trial were tested for haemagglutination-inhibition (HI) antibodies against the virus strain used in the trial or one antigenically indistinguishable from it. A four-fold or greater increase in HI titre was regarded as significant.

\section{Results}

Production and preliminary testing of 1972 recombinants

Figure 1 outlines the isolation and cloning of four recombinants. One of these (WRL 49) contained the haemagglutinin of $\mathrm{A} /$ England/42/72 and the neuraminidase of $\mathrm{A} / \mathrm{Okuda} / 57$, which is further evidence that recombination occurred in this experiment. Recombinants WRL 55, 64 and 68 were potential vaccine strains as they had the growth characteristics in eggs of $\mathrm{A} / \mathrm{Okuda} / 57$ and the external antigens of $\mathrm{A} /$ England $/ 42 / 72$, and these recombinants were examined for attenuation in quarantined volunteers. The results are summarized in Table 1 . The figures in parentheses in the first row of this table are the total number of volunteers with an initial serum HI titre of $1 / 24$ tested with each virus, usually in several experiments. Other volunteers whose initial serum HI titres were somewhat greater $(1 / 24$ to $1 / 96$ or occasionally higher) were also included in the tests. Analysis of the results showed no significant differences between those obtained in volunteers with different initial levels of antibody and so the results have been combined for simplicity of presentation in Tables 1, 2 and 3.

No tests were carried out in volunteers with the actual strain of $\mathrm{A} /$ England/42/72 used in our recombination experiment. Thus, we have no proof that attenuation was achieved by recombination with the A/Okuda/57 strain. The results shown in Table 1 refer to A/England/42/72 strain which was obtained from the same source as that used in the recombination studies but which had received three additional egg passages in another laboratory before it was tested in volunteers. This virus was attenuated in terms of its clinical reaction and clinical score when tested in February, 1974. It should, however, be pointed out that it was very difficult at this time to find susceptible volunteers, and even some of those with apparently low levels of initial antibody failed to respond to challenge. In contrast, the recombinant WRL 55 was tested during the early part of 1973 and the recombinants WRL 65 and 68 in middle and late 1973 and volunteers responded more consistently. Nevertheless, from the experience summarized in Table 1, the recombinants, in fact, are believed to have been attenuated; indeed, in view of later experience with this virus in isolated communities (Hilary, Dowling and Freestone, unpublished results),

TABLE 1. Volunteer trials of 1972 recombinants

\begin{tabular}{lcccc}
\hline & \multicolumn{4}{c}{ Viruses* } \\
\cline { 2 - 5 } & A/E/42/72 & WRL 55 & WRL 65 & WRL 68 \\
\hline No. of volunteers $\dagger$ & $11(4)$ & $35(30)$ & $13(4)$ & $13(5)$ \\
Initial HI titre (mean) & 58 & 11 & 42 & 45 \\
Clinical score (mean) $\ddagger$ & $6 \cdot 0$ & $4 \cdot 4$ & $3 \cdot 5$ & $6 \cdot 2$ \\
Virus excretion $(\%) \ddagger$ & 45 & 23 & 23 & 31 \\
Sero-conversions $(\%) \ddagger$ & 55 & 60 & 54 & 38 \\
Conclusion§ & $?$ & att & att & att \\
\hline
\end{tabular}

* See text for origin of viruses.

$\uparrow$ Total number of volunteers used over several trials (initial HI titre $<1 / 24$ to $1 / 96$ );

the figure in parenthesis is the number of $\mathrm{HI}$ seronegative $(<1 / 24)$ volunteers $)$.

$\ddagger$ See Materials and Methods for explanation.

$\S$ att = attenuated; ? = inconclusive result. 
TABLE 2. Stability of WRL 55 strain

\begin{tabular}{lccc}
\hline & \multicolumn{3}{c}{ Passage level* } \\
\cline { 2 - 4 } & E1 $\left(10^{6.5} \mathrm{EID}_{50}\right)$ & $\mathrm{E} 1 \mathrm{Hu} 1\left(10^{3} \mathrm{EID}_{50}\right)$ & $\mathrm{E} 1 \mathrm{Hu}\left(10^{2} \mathrm{EID}_{50}\right)$ \\
\hline No. of volunteers $\dagger$ & $35(30)$ & $5(3)$ & $5(5)$ \\
Initial HAI titre (mean) & 11 & 18 & $6 \cdot 5$ \\
Clinical score (mean) $\ddagger$ & $4 \cdot 4$ & $3 \cdot 5$ & $1 \cdot 9$ \\
Virus excretion $(\%) \ddagger$ & 23 & 60 & 40 \\
Sero-conversions $(\%) \ddagger$ & 60 & 20 & 40 \\
Conclusion§ & att & att & att \\
\hline
\end{tabular}

* $\mathrm{E}=$ egg passage; $\mathrm{Hu}=$ human passage; the figure in parenthesis is the estimated virus dose per volunteer.

$\dagger$ Total number of volunteers used over several trials (initial HI titre 1/24 to 1/96; the figure in parenthesis is the number of $\mathrm{HI}$ seronegative $(1 / 24)$ volunteers.

$\ddagger$ See Materials and Methods for explanation.

$\S$ att $=$ attenuated.

TABLE 3. Volunteer trials of 1974 recombinants

\begin{tabular}{|c|c|c|c|c|c|}
\hline & \multicolumn{5}{|c|}{ Viruses* } \\
\hline & A/Fin/74 & WRL 100 & WRL 94 & WRL 95 & WRL 105 \\
\hline No. of volunteers $\dagger$ & $4(1)$ & $22(10)$ & $22(7)$ & $5(4)$ & $37(28)$ \\
\hline Initial HAI titre (mean) & 38 & 40 & 36 & 16 & 10 \\
\hline Clinical score (mean) $\ddagger$ & $23 \cdot 5$ & 22 & $3 \cdot 0$ & $8 \cdot 6$ & $10 \cdot 8$ \\
\hline Virus excretion $(\%)_{+}^{+}$ & 25 & 68 & 18 & 40 & 46 \\
\hline Sero conversions $(\%) \ddagger$ & 75 & 73 & 45 & 60 & 75 \\
\hline Conclusion§ & vir & vir & over att & att & att \\
\hline
\end{tabular}

* See text for origin of viruses.

† Total number of volunteers used over several trials (initial HI titre $<1 / 24$ to $1 / 96$ ); the figure in parenthesis is the number of HI seronegative $(<1 / 24)$ volunteers.

¥ See Materials and Methods for explanation.

$\S$ vir $=$ virulent; att $=$ attenuated.

and subsequent experience with the 1974 recombinants, it is considered that WRL 55 was somewhat over-attenuated for a live virus vaccine strain.

\section{Stability of 1972 vaccine strain to human passage}

There is no reliable laboratory marker which can establish attenuation of an influenza virus strain for man. Thus, repeated passage from man to man is the best way of showing that attenuation of a virus is a stable property. Such a study has been carried out with recombinant WRL 55 and the results are shown in Table 2. The first column shows results from several trials using virus which had received only a single egg passage after cloning (these results are also shown in Table 1). Nasal washings taken from the person showing the most severe reaction (clinical score 23) with this virus inoculation were given directly to five more volunteers (see second column in Table 2). In turn, nasal washings taken from the person showing the most severe reaction in that trial (clinical score 14) were given directly to a further five volunteers (last column in Table 2). There was no indication of any increase in virulence for man by this method.
Production and preliminary testing of 1974 recombi $\overrightarrow{\bar{O}}$ nants

The production and isolation of recombinants of the A/Finland/4/74 were essentially the same ass. summarized in Fig. 1. Nineteen presumptive re을 combinants were obtained and six of these had the properties of potential live vaccine strains. Foue were tested for attenuation in volunteers (Table 3 ) $\frac{3}{6}$

The parent virus (A/Finland/4/74) was tested in four volunteers (Table 3 ). Three of these had $\mathrm{HR}$ titres of between 1/48 and 1/72 and their reactions? were only mild (clinical score between 6 and 8 ). Thes reaction in the single seronegative individual ( $\mathrm{H}$ ․ㅡ. titre less than 1/6) was severe (clinical score 74) and typical of wild virulent influenza.

Only one recombinant, WRL 100 (Table 3), pro N duced reactions similar to the parental A/Finland/4/N 74 , and then only in a small number of individuals.? their clinical scores ranged from 0 to 66 with a meano value of 27 . We concluded that this virus was lesso virulent than the wild parent but still too virulent for? use as a live vaccine strain.

Recombinant WRL 94 produced extremely mild? clinical reactions (Table 3 ) even in seronegative 
individuals (range $0-17$, mean $4 \cdot 0$ ). However, as an HI antibody rise was detected in only $45 \%$ of volunteers, and virus excretion in $18 \%$ of volunteers, it was concluded that this virus was over-attenuated for use as a vaccine.

The remaining two recombinants shown in Table 3, WRL 95 and WRL 105, appeared to be satisfactorily attenuated. WRL 105 became available for further study earlier than WRL 94, and so subsequent work was concentrated on it. Even when tested in twenty-eight seronegative volunteers, WRL 105 produced only mild clinical reactions. The highest clinical score observed was 58 in a single individual and the mean value for the seronegative volunteers was 12.7. The percentage of volunteers showing virus excretion and an $\mathrm{HI}$ antibody response after inoculation with WRL 105 were similar to those infected with A/Finland/4/74 or the virulent recombinant WRL 100. The average level of virus found in nasal washings after infection with WRL 105 (calculated from the higher of the two values obtained from each volunteer-see Methods) was $10^{2 \cdot 7} \mathrm{EID}_{50} / \mathrm{ml}$ (range $10^{0 \cdot 3}$ to $10^{5 \cdot 2} \mathrm{EID}_{50} / \mathrm{ml}$ ). Only one volunteer infected with A/Finland/4/74 excreted virus and the higher of the two values obtained was $10^{4 \cdot 0} \mathrm{EID}_{50} / \mathrm{ml}$.

A formal demonstration of the stability of the attenuation of WRL 105 by serial passage in man is being planned. Meanwhile, from the results of transmission and other studies which have now been carried out in approximately 600 volunteers, there is no indication of any instability of this strain. Results from seventy of these volunteers have been analysed in detail (Moffat et al., 1976), and show that $40 \%$ excreted virus at approximately the same level as observed in the preliminary isolation trials. The clinical reactions were mild and similar to those obtained with a placebo, and an HI antibody response was detected in $80 \%$ of the volunteers following a single intranasal dose of vaccine.

\section{Discussion}

The features of the A/Okuda/57 recombination system that make it so suitable for the production of live vaccine strains are listed in Fig. 2. The first and most important is the safety of the parent virus,

\section{FEATURES OF OKUDA RECOMBINATION SYSTEM}

(1) Safe parent.

(2) Reproducibility.

(3) High frequency of attenuated recombinants.

(4) Recombinants (a) have good immunogenicity,

(b) have good growth capacity,

(c) are reasonably stable.

FIG. 2. as documented by the extensive use of the strain as a vaccine in Japan in both adults and young children. In field trials carried out between 1960 and 1966, more than 100,000 people, including school children, were vaccinated. In particular, approximately 9000 children aged between 1 and 13 years in primary schools in the Minato ward of Osaka received the vaccine in 4 consecutive years, 1961-1964. Only $1.8 \%$ of the children had a mild febrile response, although they were susceptible to the virus as shown by the fact that $92 \%$ had a significant increase in $\mathrm{HI}$ antibody (see Okuno and Nakamura, 1966 for references). Antigenically, the virus is identical to A/Singapore/1/57 $\left(\mathrm{H}_{2} \mathrm{~N}_{2}\right)$, and the majority of the human population has been exposed to virus of this sub-type.

Since attenuation could be transferred to two quite different strains, it seems that this was not the result of some special interaction between the genetic material of the attenuated strain and that of one particular wild strain, and so similar transfer should be feasible with any new influenza A virus. Suitable recombinants were found at high frequency and this is important in that, when a new wild strain appears, a suitable recombinant must be found as quickly as possible, and without the need to use too many of that precious commodity-volunteers. On the basis of the two experiments described, it seems it may only be necessary to prepare and test three or four potential vaccine recombinant strains in order to find one which is suitable.

There can be no fixed definition of what constitutes a suitable vaccine strain, as this will depend on the immune status and age of the population in which it is to be used. For example, after exposure for several years to virus of a particular antigenic type, such as at present with the $\mathbf{H}_{3}$ haemagglutinin and $\mathrm{N}_{2}$ neuraminidase, a less attenuated strain will be needed to ensure good immunogenicity (i.e. approximately $75 \%$ seroconversions) than would have been necessary in 1969, just after the first appearance of the $\mathrm{H}_{3}$ haemagglutinin.

There are some properties which are needed for any candidate vaccine strain, namely good growth in eggs, genetic stability and lack of transmissibility. One argument in favour of live influenza virus vaccines is that they can be produced more economically and quickly than killed vaccines, since a larger number of doses should be obtained from each inoculated egg. It follows that good growth capacity in the egg is extremely important. The authors' own results and those of other workers (Peetermans, Lamy and Delem, 1974) show that a dose of at least $10^{6.5} \mathrm{EID}_{50}$ per adult is required and if allowance is made for losses during storage and freeze-drying, etc., then a minimum titre of $10^{8.5} \mathrm{EID}_{50} / \mathrm{ml}$ of allantoic fluid is required to make a live vaccine practical. 
A continuing difficulty with live influenza virus vaccines is the lack of suitable laboratory markers for attenuation. At present, the genetic stability of a proposed strain can only be established by tests in human volunteers. The authors believe that the test described for the 1972 strain (WRL 55) gives a reasonable indication of genetic stability, and a similar test is now in progress for the 1974 strain. However, it remains an urgent need to determine the biochemical basis for the attenuation of live influenza virus vaccine strains, and to link this to a test which could be readily applied to a large number of isolates. Techniques in the biochemistry and biology of influenza virus are now approaching the stage where individual polypeptides and/or segments of RNA can be identified. The recombinants which have been developed should provide a good starting point for applying such techniques to the identification of those genes which control attenuation in the A/Okuda/57 recombination system.

\section{Acknowledgments}

We would like to thank Drs D. A. J. Tyrrell, N. B. Finter and A. J. Beale for their encouragement, Dr D. S. Freestone for many discussions and access to his unpublished results, and $\mathrm{Mr}$ M. Greenaway for his valuable technical assistance.

This recombination system is the subject of Patent Application No. 55469/73.

\section{References}

Beare, A.S. \& Hall, T.S. (1971) Recombinant influenza A viruses as live vaccines for man. Lancet, ii, 1271.
Beare, A.S., MaAssab, H.F., Tyrrell, D.A., Slepushiniñ A.N. \& Hall, T.S. (1971) A comparative study of attenuS ated influenza viruses. Bulletin of the World Healt Organization, 44, 593.

FazeKaS DE ST Groth, S. \& White, D.O. (1958) An im: proved assay for the infectivity of influenza viruses Journal of Hygiene, 56, 151.

Huygelen, C., Peetermans, J., Vascoboinic, E., Berge, E⿱宀 \& Colinet, G. (1973) Live attenuated influenza virus vaccine: in vitro and in vivo properties. Proceedings of the International Symposium on Influenza Vaccines for Me and Horses, London, 1972. Symposia Series in Immuno biological Standardization, 20, 282.

MaAssab, H.F., Kendal, A. \& Davenport, F. (1972) Hybrid formation of influenza virus at $25^{\circ}$. Proceedings $\not \dot{\Phi}$ the Society of Experimental Biology and Medicine, 139, 768-

McCahon, D., Schild, G.C., Beare, A.S. \& Hall, T.S.W (1973) Use of recombination in the production of influenz? vaccine strains. Postgraduate Medical Journal, 49, 195.

Moffat, M., Stealey, V.M.,Freestone, D.S. \& Macdonald A. (1976) Assessment of immunogenicity, reactivity antid. transmissibility of recombinant WRL 105 strain of live attenuated influenza vaccine. Journal of Biological Stanं dardization, 4, 2.

NAKAMURA, K. (1961) [Studies on the live vaccine of the influenza A2.] Virus II, 257 [in Japanese with Englis@ Summary].

OKUnO, Y. \& NaKamura, K. (1966) Prophylactic effectiveness of live influenza vaccine in 1965. Biken Journal, 9, 89.

Peetermans, J., Lamy, F. \& Delem, A. (1974) Immune re sponse to combined live influenza virus vaccines adminis $\overline{\underline{z}}$ tered intranasally. Developments in Biological Standardiza tion, 28, 340.

REED, L.T. \& MUENCH, H. (1938) A simple method of mating $50 \%$ end points. American Journal of Hygiene 493.

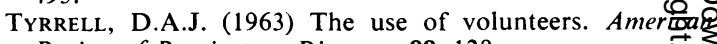
Review of Respiratory Diseases, 88, 128. 